\title{
Femoroacetabular Impingement and Abnormal Femoral Version-Treatment with Arthroscopy and Derotational Osteotomy
}

\author{
David J Kirby, MD*, Daniel B Buchalte, MD, Craig M Capeci, MD and Thomas Youm, MD \\ Department of Orthopedic Surgery, NYU Langone Health, New York, USA
}

\begin{abstract}
Femoroacetabular impingement (FAI) leads to chondral defects and labral degeneration of the hip joint which present initially with pain and functional limitation, eventually progressing to osteoarthritis. This disease process is associated with femoral version abnormalities which in and of themselves can result in aberrant joint stresses and joint degeneration. Addressing the hip joint without correcting femoral version in these cases can result in failure given the persistent joint dysfunction. Hip arthroscopy has proven an effective intervention for FAl, with outcomes on par with open treatment. Combining this surgery with a mini-open femoral osteotomy is an efficient one-stage operation for the correction of femoral version abnormalities in the setting of FAI. The use of a femoral nail for fixation of the osteotomy allows for no added postoperative weight bearing or rehabilitation requirements. Hip arthroscopy with mini-open femoral osteotomy is a safe and effective method for a one-stage treatment of this complex disease.
\end{abstract}

\section{Keywords}

Femoral anteversion, Femoral derotational osteotomy, Femoroacetabular impingement, Hip arthroscopy

\section{Introduction}

Hip arthroscopy is a popular technique for addressing intraarticular pathology [1] with ever expanding indications for surgery [2]. The growing complexity of patient pathoanatomy often requires surgical management beyond the standard hip arthroscopy patient. One such group is patients with femoroacetabular impingement (FAl) and concomitant abnormal femoral version.

Studies related FAl and femoral version have found that almost half of patients with symptomatic FAl have femoral version abnormalities, with $12 \%$ having femoral version greater than 35 degrees [3]. Unfortunately, abnormal femoral version is a predictor for poorer outcomes in patients undergoing hip arthroscopy [4]. Excessive femoral retroversion (< 5 degrees) or anteversion ( $>25$ degrees) results in increased anterior joint stress and gait abnormalities [5-7]. Over time these lead to the joint damage and can progress to osteoarthritis [8].

Management of this condition continues to be optimized, with surgical techniques focused on minimizing morbidity and improving overall outcomes. The initial treatment algorithm for these patients follows the standard conservative routine, including activity modification, nonsteroidal anti-inflammatory drugs, and physical therapy. Patients who fail conservative management and have a clinical examination and imaging consistent with FAI and abnormal femoral version can be considered for hip arthroscopy with open derotational osteotomy. We present a method for arthroscopic hip labralrepair using suture anchors with open derotational femoral osteotomy.

\section{Surgical Technique}

\section{Preoperative assessment}

Primary complaints on presentation are typically attributable to FAI (groin or hip pain, clicking/popping with hip flexion) while femoral anteversion may be asymptomatic or the patient could complain of intoeing. On examination, patients will demonstrate limited hip flexion and pain with impingement testing. Femoral anteversion can be estimated with the

*Corresponding author: David J Kirby, MD, Department of Orthopedic Surgery, NYU Langone Health, 301 East $17^{\text {th }}$ Street, New York, NY 10003, USA, Tel: (212)-598-6000

Accepted: September 23, 2020

Published online: September 25, 2020

Citation: Kirby DJ, Buchalte DB, Capeci CM, et al. (2020) Femoroacetabular Impingement and Abnormal Femoral Version-Treatment with Arthroscopy and Derotational Osteotomy. J Orthop Surg Tech 3(2):158-163 


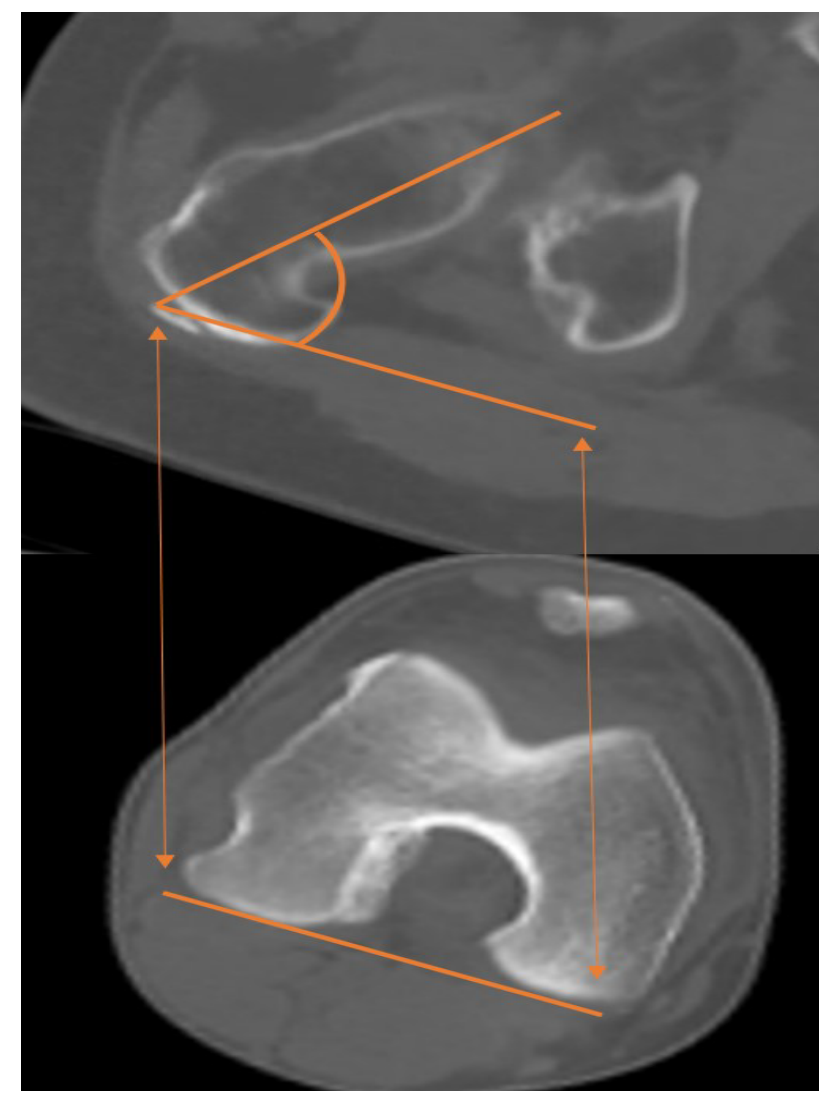

Figure 1: Femoral version measurements are calculated from axial computerized tomography (CT) images as the angular difference between the line created by the posterior most aspect of the distal femoral condyles and the line of the femoral neck. Normal femoral version is $10-25$ degrees.

trochanteric prominence angle test [9]. Workup begins with plain film radiographs to evaluate for cam/pincer lesions and acetabular retroversion. Magnetic resonance imaging (MRI) evaluates for labral integrity and cartilage defects. If abnormal femoral version is suspected, a computerized tomography (CT) from the hip through the knee measures femoral version as the angular difference between the posterior condylar axis of the distal femur and the axis of the femoral neck (Figure 1). Initial management should include 6 months of nonoperative care, with a focus on physical therapy. Patients who remain symptomaticafter 6 months of conservative treatment, with imaging confirmation of FAI abnormalities and femoral anteversion of $>30$ degrees, are indicated for hip arthroscopy and open derotational femoral osteotomy. Patients with severe articular cartilage damage are contraindicated for surgery. (Table 1) summarizes the indications and contraindications for surgery.

\section{Positioning}

The patient is positioned supine on a traction table (Figure 2a). An exam under anesthesia is performed to evaluate for internal and external rotation with the hip in neutral and flexed to 90 degrees. A well-padded post is placed in the perineal region. The ipsilateral arm is forward flexed and secured across the patient's chest with the elbow flexed to remove it from the operative field. The contralateral leg is abducted

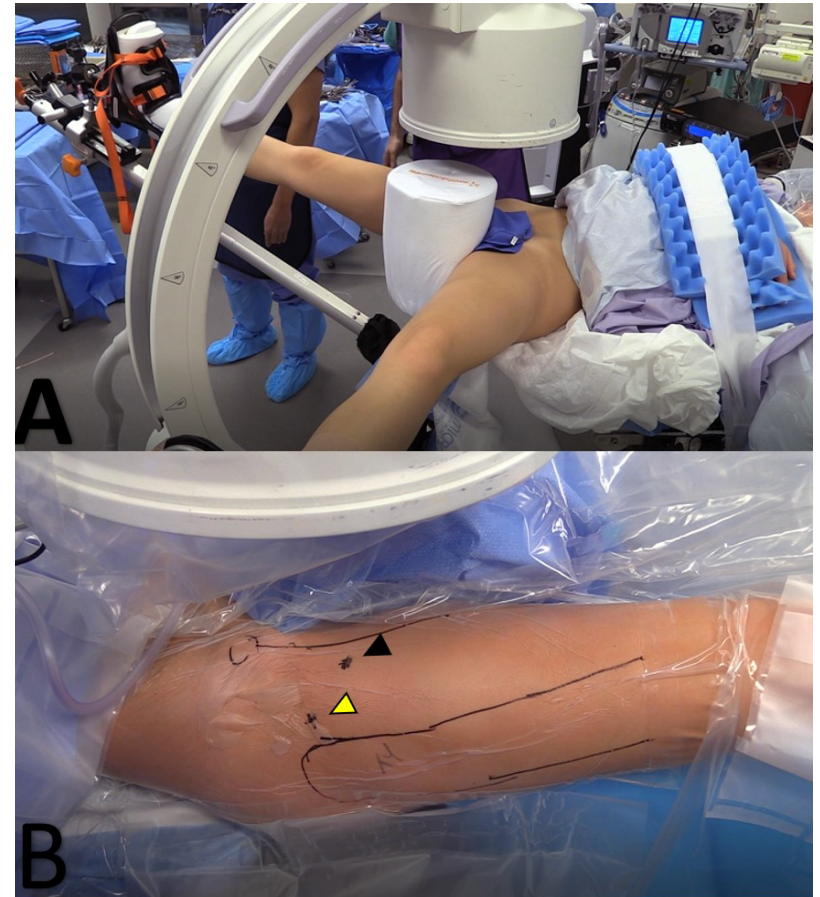

Figure 2: Patient positioning (A) and draping (B) are shown. (A) Patients are typically positioned supine on a traction table with the non-operative leg abducted enough to allow for adequate C-arm imaging; (B) A surgical field from the iliac crest proximally to the knee distally is prepped out. The standard anterolateral (yellow arrow) and mid-anterior (black arrow) portals are used to perform the hip arthroscopy.

30 degrees. Traction is then applied to the operative leg with slight adduction of the ipsilateral hip (5-10 degrees) to assist with joint distraction. A large C-arm is then brought in from the non-operative side between the legs and positioned to allow for appropriate intraoperative hip imaging. It is important to drape out from the iliac crest proximally to the knee distally to allow for hip arthroscopy and femoral osteotomy.

\section{Surgical technique}

Standard diagnostic arthroscopy is performed using anterolateral and mid anterior portals (Figure $2 b$ ). Briefly, chondral surfaces and the labrumare inspected for defects. The acetabular rim and subspine area should be inspected, where impingement is noted and resected. If appropriate, labral tear is repaired with \#2 flat-braided sutures secured using 2.9 $\mathrm{mm}$ knotless suture anchors as previously described [10]. Decreased femoral head-neck offset anterolaterally is addressed with femoral neck osteochondroplasty is performed with a 5.5 bur. Capsular laxity, as denoted with supraphysiologic distraction of the femoral head with traction, can be addressed with capsular imbrication [10].

At this point, the C-arm is used to locate a proximal starting point for intramedullary nailing of the femur. A lateral approach to the hip used, with a 3-4 cm incision made 3-4 $\mathrm{cm}$ proximal to the tip of the greater trochanter (Figure 3a). The fascia lata is sharply incised slightly distal to the skin incision, in line with the expected trajectory of nail insertion. Muscle should be bluntly spread to obtain direct contact on 
Citation: Kirby DJ, Buchalte DB, Capeci CM, et al. (2020) Femoroacetabular Impingement and Abnormal Femoral Version-Treatment with Arthroscopy and Derotational Osteotomy. J Orthop Surg Tech 3(2):158-163

Table 1: Indications and contraindications.

\begin{tabular}{|l|l|}
\hline Indication & Contraindication \\
\hline Symptoms for greater than 6 months with failed trial of conservative treatment & Severe articular cartilage damage \\
Imaging evidence of FAl & \\
Severe anteversion (> 30 degrees) & \\
\hline
\end{tabular}

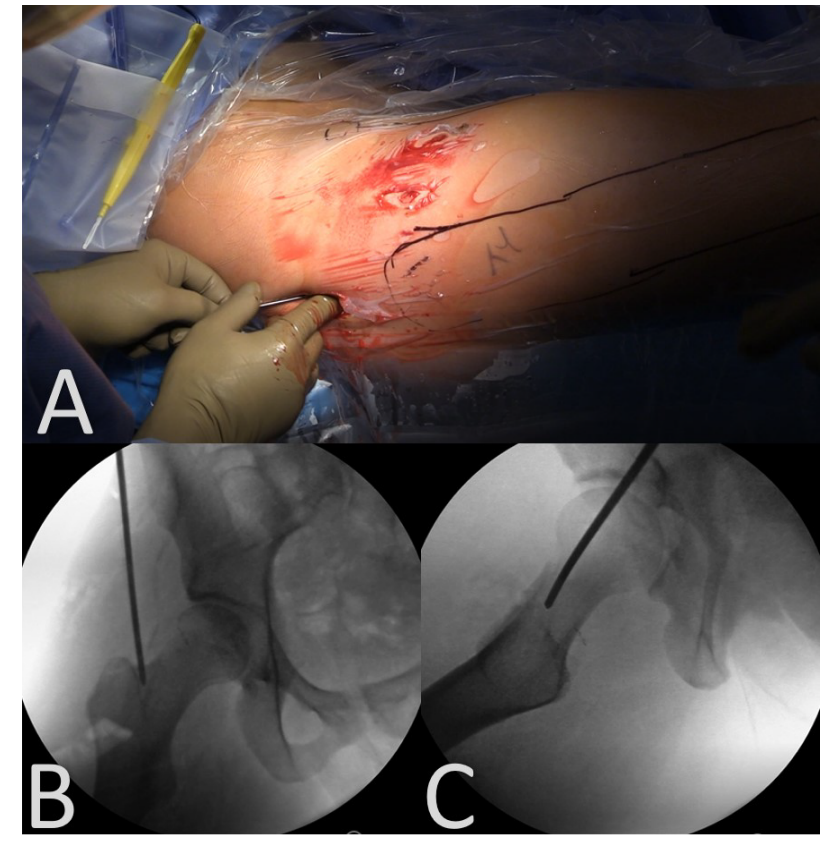

Figure 3: (A) Clinical imaging and $X$-rays demonstrating appropriate insertion trajectory for the intramedullary nail. The skin incision should be $3-4 \mathrm{~cm}$ proximal to the tip of the greater trochanter; ( $B$ and $C$ ) The guidewire for the femoral nail should be at the medial tip of the greater trochanter, in-line with the medullary canal.

bone. The guidewire is then inserted, with the starting point on the medial tip of the greater trochanter, in line with the center of the medullary canal. The wire is checked on both $\mathrm{AP}$ and lateral views on the $\mathrm{C}$-arm to confirm location (Figure $3 b$ and Figure $3 c$ ). The guidewire can then be drilled to the level of the lesser trochanter. A $12-15 \mathrm{~mm}$ opening reamer is used to create the proximal entry site for the nail. When using the opening reamer, it is important to use the soft tissue protector to protect the hip abductors. The opening reamer and guide wire can then be removed as a unit. A balltip guidewire is inserted into the medullary canal and advanced to the distal femoral physeal scar. Nail length is determined by measuring the length of the inserted balltip wire with a radiolucent ruler. Fluoroscopy is used to confirm the ruler is flush with bone. Next, sequential reaming starts with a $9 \mathrm{~mm}$ reamer and continues at $0.5-1 \mathrm{~mm}$ increments up to $1.5 \mathrm{~mm}$ above the intended nail diameter. In the highlighted case, this means reaming to $11.5 \mathrm{~mm}$ for a $10 \mathrm{~mm}$ nail.

The femoral osteotomy is performed next. The balltip guide wire is left inserted during the osteotomy to maintain the femoral reduction. Steinman pins are inserted percutaneously proximally in the subtrochanteric region and distally in the metadiaphysis to guide in the rotational correct. The

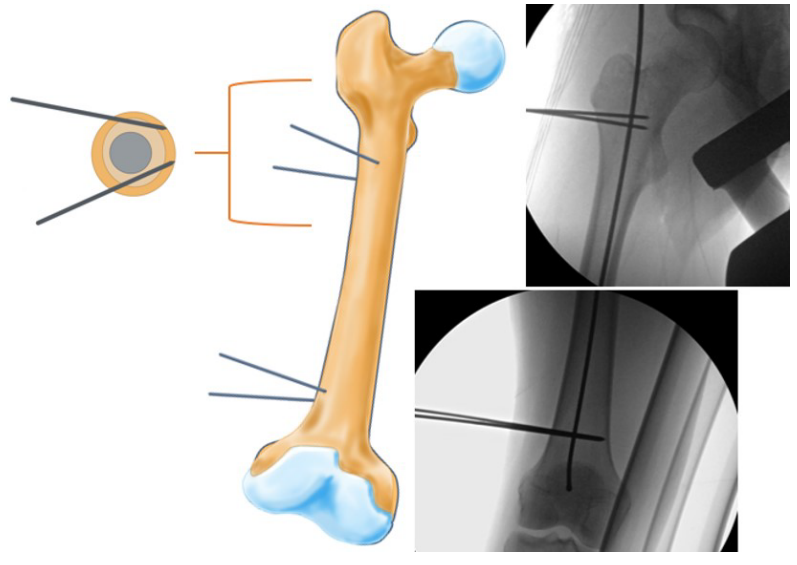

Figure 4: Steinman pins are placed in the subtrochanteric region and the distal metadiaphyseal region, which will aid in rotational correction. The Steinman pins should be placed in a manner as to allow for passage of the intramedullary nail, as shown in the illustration.

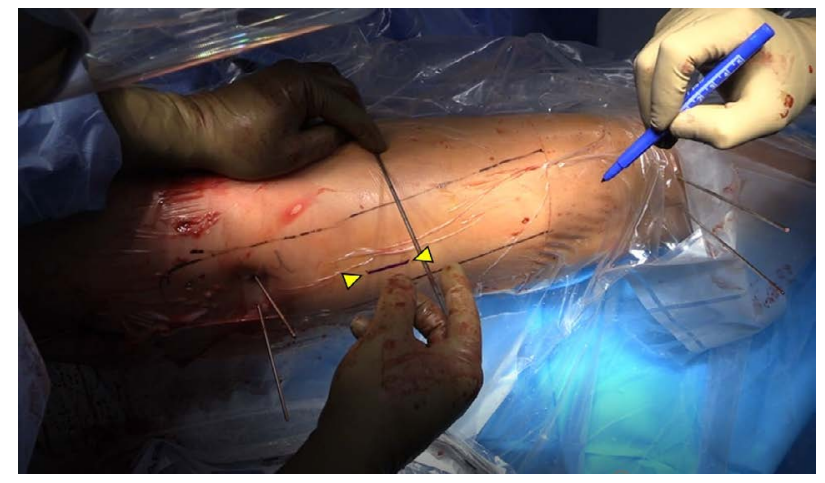

Figure 5: The lateral approach to the femur is utilized to perform the open femoral osteotomy (yellow arrows). This incision is typically $3 \mathrm{~cm}$ in length and is roughly $6 \mathrm{~cm}$ distal to the lesser trochanter.

pins are inserted at a parallel trajectory on an anteroposterior view but converging on an axial perspective to allow for passage of the future nail (Figure 4). The angular difference between proximal and distal anterior Steinman pins should be noted to guide future correction. Next, a 3-4 cm skin incision is made on the lateral thigh centered $5-6 \mathrm{~cm}$ distal to the lesser trochanter for the femoral osteotomy (Figure 5). The fascia lata is sharply split and the vastus lateralis elevated to expose the femur via the intermuscular septum. A $4.5 \mathrm{~mm}$ drill is used to create multiple drill holes in an attempt to osteotomize the medial femoral cortex. A sagittal saw is used to osteotomize the lateral femoral cortex (Figure 6). Any remaining intact medial cortex is osteotomized with an osteotome. 


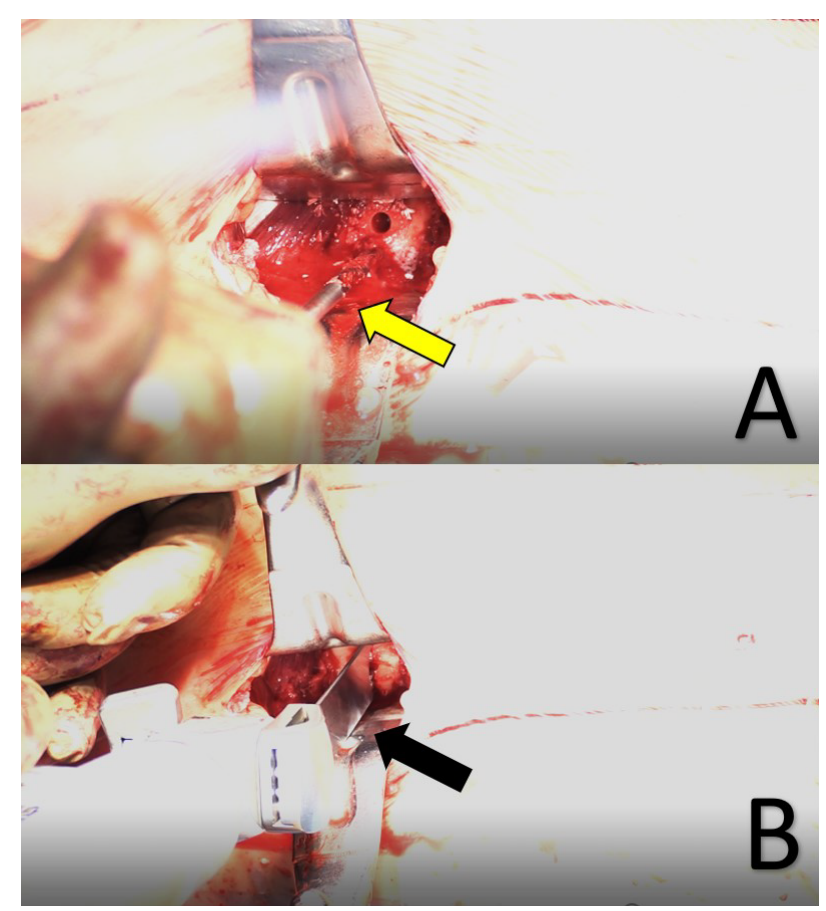

Figure 6: (A) The femoral osteotomy is performed from a lateral approach. A $4.5 \mathrm{~mm}$ drill (yellow arrow) is first used to create multiple drill holes converging on the medial cortex to perform a medial osteotomy; (B) A sagittal saw (black arrow) is then used to osteotomize the lateral cortex of the femur.

Once the osteotomy is completed, the distal femur was rotated externally to correct the abnormal version, with a goal anteversion of 15 degrees. A sterile goniometer is used to confirm the degree of correction (Figure 7). Next, the intramedullary nail is inserted. During nail insertion care must be taken to maintain the corrected femoral rotation. Clamps are used to hold the proximal and distal femoral segments aligned with regular checking with the goniometer. Once the nail is seated and femoral rotation is confirmed, the ballpin guide wire can be removed and distal locking screws placed. The nail is then backslapped for compression across the osteotomy site and proximal locking screws are placed. Once again, the goniometer is checked to confirm adequate correction. Fluoroscopic images are taken to confirm nail position. The wounds are copiously irrigated and closed. Post-operative examination under anesthesia is performed to confirm the version correction.

\section{Postoperative protocol}

The postoperative rehabilitation protocol is broken into 3 phases. In Phase I (weeks 0-4), patients are made flat-foot weight bearing with two crutches and are kept in a hip brace for 1-4 weeks to prevent rotation/abduction. No external rotation, hyperextension, or flexion with abduction are allowed, however flexion is allowed as tolerated. Therapy will focus on normalizing the gait pattern with additional exercises of stationary bike for 20 minutes per day, supine hip log rolling for internal rotation, pelvic tilts, and progressive range of motion with stool rotation for assisted active hip internal rotation. During the first 2 weeks, patients are also prescribed $81 \mathrm{mg}$ of

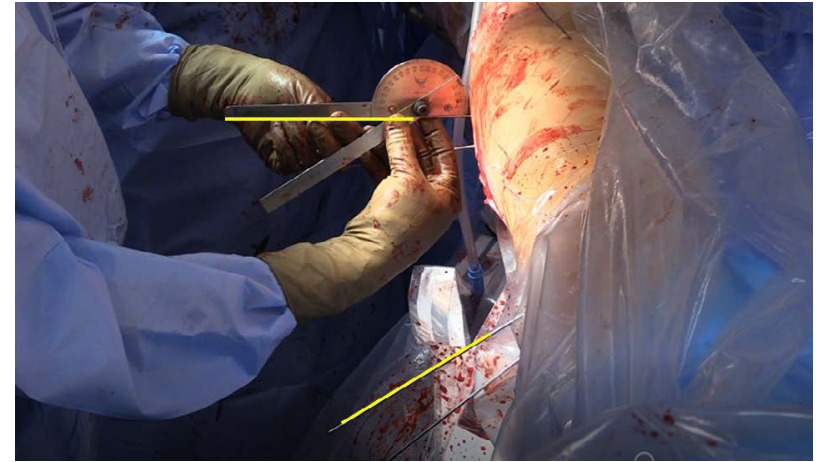

Figure 7: The rotational correction is confirmed with a sterile goniometer using the Steinman pins (yellow lines) as a reference for femoral alignment.

aspirin twice a day for deep vein thrombosis prophylaxis. In Phase II (weeks 4-8), patients are advanced to weightbearing as tolerated and weaned off crutches. Patients can progress to range of motion as tolerated with proprioceptive training and progressive strengthening with hip flexion isotonics, leg press exercise, and isokinetics. In phase III (8+ weeks), patients continue to advance in strength training and by 3-6 months patients perform treadmill running and sport specific agility drills. Return to sport can be expected at 6 months.

\section{Discussion}

Hip arthroscopy results for treating labral tears from FAI are known to be excellent [11-13]. Similarly, femoral derotational osteotomies for treating femoral version abnormalities have excellent results [14]. Despite excellent results individually, to our knowledge, no analyses have been performed looking at the results of treating both FAl and femoral version abnormalities simultaneously. However, we do know that untreated femoral version abnormalities make the arthroscopic treatment of FAl less likely to be successful [8,15-17]. It is hypothesized that untreated abnormal femoral version in the setting of hip arthroscopy may lead to increased stress on the anterior acetabulum and anterior soft tissue structures of the hip, leading to impingement and repair failure $[4,8,16$ 20]. Simply correcting the sequelae of these abnormalities, namely a cam/pincer lesion and labral tear, does nothing to address the source of these problems. For this reason, we have begun to treat patients who have FAl and femoral version abnormality with arthroscopic cam/pincer treatment, labral repair, and open femoral osteotomy. While indications for these simultaneous procedures are not concrete, senior author T.Y. performs these surgeries on patients with severe anteversion ( $>30$ degrees), pain with flexion, adduction, and internal rotation, failed 6 months of conservative treatment, and structural evidence of impingement.

While many techniques for performing labral repair and cam/pincer resection have been described and validated, surgeons continue to argue about the best way to perform femoral derotational osteotomies. Technical advances such as proximal versus distal osteotomies, different fixation devices such as ilizarov frames, intramedullary devices, and 
Citation: Kirby DJ, Buchalte DB, Capeci CM, et al. (2020) Femoroacetabular Impingement and Abnormal Femoral Version-Treatment with Arthroscopy and Derotational Osteotomy. J Orthop Surg Tech 3(2):158-163

plates, and open versus closed approaches all appear to have decreased complication rates and have good outcomes in the literature [14,21-29]. Intramedullary devices allow for immediate post-operative weight bearing and are the preferred device if a pure rotational deformity is being address without the need for coxa vara or coxa valga correction [14].

The ability to treat intra-articular hip pathology and optimize femoral version in one procedure may be ideal, though studies supporting its use are limited. As discussed by Hartigan, et al. [23], performing a femoral derotational osteotomy in addition to hip arthroscopy would likely correct the underlying version abnormality leading to FAI, correct the FAl, improve preoperative anterior instability, and improve hip internal and external rotation abnormalities. However, disadvantages of a simultaneous procedure include a longer operation, longer recovery, and increased hardware. With relatively few disadvantages, simultaneous treatment of femoral version abnormalities and FAI, being able to reduce the re-development of FAI and thus osteoarthritis, appears to be an ideal treatment for patients like the one described in this technique guide. More cases and rigorous analyses are required prior to definitively stating the benefit of these joint procedures, but early case reports seem to be promising $[14,23]$. When enough simultaneous hip arthroscopies and femoral derotational osteotomies are performed, we will be able to further refine our indications, and identify patients who are at higher risk for failure of isolated hip arthroscopy for FAl.

\section{Disclosures}

Dr. Youm is a paid consultant for Arthrex and Smith \& Nephew. The remaining authors have nothing to disclose.

\section{Authorship Declaration}

All authors meet authorship criteria and agree with the manuscript.

\section{References}

1. Sing DC, Feeley BT, Tay B, et al. (2015) Age-related trends in hip arthroscopy: A large cross-sectional analysis. Arthroscopy 31: 2307-2313.

2. Stevens MS, Legay DA, Glazebrook MA, et al. (2010) The evidence for hip arthroscopy: Grading the current indications. Arthroscopy 26: 1370-1383.

3. Lerch TD, Todorski IAS, Steppacher SD, et al. (2018) Prevalence of femoral and acetabular version abnormalities in patients with symptomatic hip disease: A controlled study of 538 hips. Am J Sports Med 46: 122-134.

4. Fabricant PD, Fields KG, Taylor SA, et al. (2015) The effect of femoral and acetabular version on clinical outcomes after arthroscopic femoroacetabular impingement surgery. J Bone Joint Surg Am 97: 537-543.

5. Eckhoff DG, Montgomery WK, Kilcoyne RF, et al. (1994) Femoral morphometry and anterior knee pain. Clin Orthop Relat Res 302: 64-68.

6. MacWilliams BA, McMulkin ML, Davis RB, et al. (2016) Biomechanical changes associated with femoral derotational osteotomy. Gait Posture 49: 202-206.
7. Satpathy J, Kannan A, Owen JR, et al. (2015) Hip contact stress and femoral neck retroversion: A biomechanical study to evaluate implication of femoroacetabular impingement. J Hip Preserv Surg 2: 287-294.

8. Tonnis D, Heinecke A (1999) Acetabular and femoral anteversion: Relationship with osteoarthritis of the hip. J Bone Joint Surg Am 81: 1747-1770.

9. Souza RB, Powers CM (2009) Concurrent criterion-related validity and reliability of a clinical test to measure femoral anteversion. J Orthop Sports Phys Ther 39: 586-592.

10. Shenoy K, Dai AZ, Mahure SA, et al. (2017) Arthroscopic repair of hip labrum with suture anchors. Arthrosc Tech 6: e2143-e2149.

11. Fabricant PD, Heyworth BE, Kelly BT (2012) Hip arthroscopy improves symptoms associated with FAI in selected adolescent athletes. Clin Orthop Relat Res 470: 261-269.

12. Gupta A, Redmond JM, Stake CE, et al. (2016) Does primary hip arthroscopy result in improved clinical outcomes?: 2-year clinical follow-up on a mixed group of 738 consecutive primary hip arthroscopies performed at a high-volume referral center. Am J Sports Med 44: 74-82.

13. Khan M, Habib A, De Sa D, et al. (2016) Arthroscopy up to date: Hip femoroacetabular impingement. Arthroscopy 32: 177-189.

14. Buly RL, Sosa BR, Poultsides LA, et al. (2018) Femoral derotation osteotomy in adults for version abnormalities. J Am Acad Orthop Surg 26: e416-e425.

15. Larson CM, Ross JR, Stone RM, et al. (2016) Arthroscopic management of dysplastic hip deformities: Predictors of success and failures with comparison to an arthroscopic FAl cohort. Am J Sports Med 44: 447-453.

16. Li H, Wang Y, Oni JK, et al. (2014) The role of femoral neck anteversion in the development of osteoarthritis in dysplastic hips. Bone Joint J 96: 1586-1593.

17. Fabricant PD, Bedi A, De La Torre K, et al. (2012) Clinical outcomes after arthroscopic psoas lengthening: The effect of femoral version. Arthroscopy 28: 965-971.

18. Ferro FP, Ho CP, Briggs KK, et al. (2015) Patient-centered outcomes after hip arthroscopy for femoroacetabular impingement and labral tears are not different in patients with normal, high, or low femoral version. Arthroscopy 31: 454-459.

19. Jackson TJ, Lindner D, El Bitar YF, et al. (2015) Effect of femoral anteversion on clinical outcomes after hip arthroscopy. Arthroscopy 31: 35-41.

20. Chaharbakhshi EO, Hartigan DE, Perets I, et al. (2019) Is hip arthroscopy effective in patients with combined excessive femoral anteversion and borderline dysplasia? A match-controlled study. Am J Sports Med 47: 123-130.

21. Moens P, Lammens J, Molenaers G, et al. (1995) Femoral derotation for increased hip anteversion. A new surgical technique with a modified Ilizarov frame. J Bone Joint Surg Br 77: 107-109.

22. Hinterwimmer S, Minzlaff $P$, Saier T, et al. (2014) Biplanar supracondylar femoral derotation osteotomy for patellofemoral malalignment: The anterior closed-wedge technique. Knee Surg Sports Traumatol Arthrosc 22: 2518-2521.

23. Hartigan DE, Perets I, Walsh JP, et al. (2017) Femoral derotation osteotomy technique for excessive femoral anteversion. Arthrosc Tech 6: e1405-e1410.

24. Winquist RA (1986) Closed intramedullary osteotomies of the femur. Clin Orthop Relat Res 212: 155-164. 
Citation: Kirby DJ, Buchalte DB, Capeci CM, et al. (2020) Femoroacetabular Impingement and Abnormal Femoral Version-Treatment with Arthroscopy and Derotational Osteotomy. J Orthop Surg Tech 3(2):158-163

25. Chapman ME, Duwelius PJ, Bray TJ, et al. (1993) Closed intramedullary femoral osteotomy. Shortening and derotation procedures. Clin Orthop Relat Res 287: 245-251.

26. Stahl JP, Alt V, Kraus R, et al. (2006) Derotation of post-traumatic femoral deformities by closed intramedullary sawing. Injury 37 : 145-151.

27. Kamath AF, Ganz R, Zhang H, et al. (2015) Subtrochanteric os- teotomy for femoral mal-torsion through a surgical dislocation approach. J Hip Preserv Surg 2: 65-79.

28. Pailhe R, Bedes L, Sales de Gauzy J, et al. (2014) Derotational femoral osteotomy technique with locking nail fixation for adolescent femoral antetorsion: Surgical technique and preliminary study. J Pediatr Orthop B 23: 523-528.

29. Putz C, Wolf SI, Geisbusch A, et al. (2016) Femoral derotation osteotomy in adults with cerebral palsy. Gait Posture 49: 290-296.

DOI: $10.36959 / 453 / 540$

Copyright: (C) 2020 Kirby DJ, et al. This is an open-access article distributed under the terms of the Creative Commons Attribution License, which permits unrestricted use, distribution, and reproduction in any medium, provided the original author and source are credited. 